\title{
Drag Reduction in Bubbly Taylor-Couette Turbulence
}

\author{
Thomas H. van den Berg, ${ }^{1, *}$ Stefan Luther, ${ }^{1}$ Daniel P. Lathrop, ${ }^{2, \dagger}$ and Detlef Lohse ${ }^{1, \$}$ \\ ${ }^{1}$ Department of Applied Physics, University of Twente, P.O. Box 217, 7500 AE Enschede, The Netherlands \\ ${ }^{2}$ Institute for Research in Electronics and Applied Physics, University of Maryland, College Park, Maryland 20742, USA
}

(Received 21 September 2004; published 31 January 2005)

In Taylor-Couette flow the total energy dissipation rate and therefore the drag can be determined by measuring the torque on the system. We do so for Reynolds numbers between $\operatorname{Re}=7 \times 10^{4}$ and $\operatorname{Re}=$ $10^{6}$ after having injected (i) small bubbles $(R=1 \mathrm{~mm})$ up to a volume concentration of $\alpha=5 \%$ and (ii) buoyant particles $\left(\rho_{p} / \rho_{l}=0.14\right)$ of comparable volume concentration. In case (i) we observe a crossover from little drag reduction at smaller Re to strong drag reduction up to $20 \%$ at $\operatorname{Re}=10^{6}$. In case (ii) we observe at most little drag reduction throughout. Several theoretical models for bubbly drag reduction are discussed in view of our findings.

DOI: 10.1103/PhysRevLett.94.044501

PACS numbers: 47.27.-i, 47.55.Dz, 83.60.Yz

While turbulent drag reduction by polymers has fascinated the physics community for some time [1-4], it is less known that also a relatively small amount of bubbles can considerably reduce the drag [5-8]. This drag reduction method is feasible in application to ships [9]. For example, in Ref. [5] 80\% drag reduction was achieved by injecting microbubbles in the turbulent boundary layer of a submerged body. Under more realistic conditions for ships, drag reduction of about $32 \%$ has been achieved [10]. This could lead to substantial fuel saving for commercial ships.

While it is understood that the drag reduction effect is due to the modification of the turbulent boundary layer through the bubbles, a detailed and accepted explanation of the effect is lacking. Recently, numerical simulations succeeded in qualitatively reproducing drag reduction [1115]. From these simulations several different theoretical explanations of the effect arose: Ferrante and Elghobashi [12] performed a simulation with point bubbles (corresponding to a bubble radius of $R_{b} \simeq 3.2 \times 10^{-3} y_{0}$ ) in a turbulent boundary layer (of inner length scale $y_{0}$ ). The inhomogeneous bubble distribution leads to an effective compressibility of the flow which induces an enhanced average flow off the wall. This flow pushes the dissipative structures further away from the wall, leading to a net drag reduction. This suggested mechanism gets less effective for larger bubbles (at a given volume concentration $\alpha$ ). Xu et al. [11] found in direct numerical simulations for relatively large bubbles and void fractions up to $8 \%$ a transient drag reduction as bubbles disperse into the flow. Sugiyama et al. [15] extended the model of Ref. [11] but found skin friction enhancement for rigid bubbles. Only by allowing for bubble deformation within a front-tracking scheme [16] a skin friction reduction up to $3 \%$ was found at large Reynolds numbers, whereas at small Reynolds number the drag was again enhanced [15]. Also Lu et al. [13] did simulations with up to 16 deformable and much larger bubbles than employed in Ref. [12] (54 wall unit diameter) in a "minimal turbulent channel" $(\operatorname{Re}=4000)$, again employing the front-tracking technique [16]. They find that the deformable bubbles approaching the wall push high vorticity regions away from the wall, which leads to less drag. In their simulations Lu et al. [13] find drag reduction up to $15 \%$. In contrast, nondeformable buoyant particles of equal size and buoyancy ratio as the bubbles can lead to a drag enhancement $[13,15]$. Note that within the Ferrante-Elghobashi picture buoyant microparticles with the same density ratio as bubbles would also lead to drag reduction, as they imply an effective compressibility, too.

In order to shed more light on the issue of turbulent drag reduction, we performed experiments in turbulent bubble and buoyant particle Taylor-Couette flow. One advantage of the Taylor-Couette system is that statistical stationary states can be achieved easily. The second advantage of Taylor-Couette flow is that it is a closed flow system for which exact global energy balances hold. The total dissipation rate $\epsilon$ can directly be measured by measuring the required torque $T$ on the system (to keep the inner cylinder at constant velocity), namely,

$$
\epsilon=\frac{T \Omega}{2 \pi \rho L\left(b^{2}-a^{2}\right)}=\frac{\nu^{2} G \Omega}{2 \pi\left(b^{2}-a^{2}\right)},
$$

where $G=T / \rho \nu^{2} L$ is the dimensionless torque, $\rho=$ $\rho_{0}(1-\alpha)$ is the fluid density of the bubbly liquid, $\rho_{0}$ is the fluid density of water, $L$ is the length of the cylinders, $a$ and $b$ are the inner and the outer cylinder radius, respectively, and $\Omega$ is the angular rotation rate. The kinematic viscosity $\nu$ is for the bubbly liquid, which we assume follows [17]

$$
\nu=\nu_{0}\left(1+\frac{5}{2} \alpha\right)
$$

with $\nu_{0}$ the viscosity of water. The relation (2) holds for small gas volume fractions and becomes more and more questionable for larger $\alpha$, as discussed, e.g., in Ref. [18]. Here the relation (2) is needed only to define the flow Reynolds number as $\operatorname{Re}=\Omega a(b-a) / \nu$. 
To quantify the drag we take the drag coefficient which is the dimensionless energy dissipation rate $c_{\epsilon}=\epsilon(b-a) /$ $U^{3}=T /\left(2 \pi \rho L a^{3} \Omega^{2}\right)$, where $U=a \Omega$ is the velocity of the inner cylinder. Note that the drag coefficient $c_{\epsilon}$ follows directly from the measured torque $T$ and $\Omega$, without making any assumption on the viscosity $\nu$ of the bubbly flow.

The experimental setup consists of a rotating inner cylinder of radius $a=16 \mathrm{~cm}$ and a stationary outer cylinder of radius $b=22 \mathrm{~cm}$. The total length of the inner cylinder is $L=69.5 \mathrm{~cm}$ giving an aspect ratio $\Gamma=$ $L /(b-a)=11.5$. The inner cylinder consists of three parts, with the torque measured on the middle part of the inner cylinder. The torque on the middle section is measured using strain gauges. The basic experimental setup is discussed in more detail in Refs. [19-21]. Bubbles are injected by eight needles located at the lower part of the outer cylinder. The bubble size is dictated by the shear in the setup with stronger shear favoring slightly smaller bubbles [22]. Typical bubble diameters are between 0.5 and $2 \mathrm{~mm}$. The typical boundary layer inner length scale is much smaller, $y_{0}=\nu / u_{*} \approx 3 \mu \mathrm{m}$ with $u_{*}=\sqrt{\tau_{w} / \rho}$. Here, as in Ref. [19], we have estimated $\tau_{w}$ as $\tau_{w}=$ $T / 2 \pi a^{2} L$.

An outlet is attached to the top plate which leads to an expansion barrel. By simultaneously measuring the weight difference (therefore volume difference) of the barrel, we are able to measure the volume averaged void fraction $\alpha$ of the setup, as injecting bubbles in the flow forces liquid to flow out on top.

For several constant Reynolds numbers we increased the void fraction and measured the torque on the inner cylinder as well as the rotation rate of the inner cylinder and the void fraction. Our Reynolds number range spans $7 \times 10^{4}<$ $\operatorname{Re}<10^{6}$ which is limited by the maximum available torque of the motor.

In Fig. 1 we show the drag coefficient $c_{\epsilon}$ vs Re for various bubble volume concentrations $\alpha$ up to $5 \%$. We see from Fig. 1 that the drag coefficient decreases for increasing $\alpha$. To be able to better compare the drag coefficients $c_{\epsilon}(\alpha, \mathrm{Re})$ and $c_{\epsilon}(\alpha=0, \mathrm{Re})$, we plot their ratio as a function of Re in Fig. 2. Rather than using the individual data points $c_{\epsilon}(\alpha=0, \mathrm{Re})$, the ratio is calculated by first fitting the data points for $c_{\epsilon}(\alpha=0, \operatorname{Re})$ to [23]

$$
c_{\epsilon}^{\mathrm{fit}}(\alpha=0, \mathrm{Re})=c_{\epsilon, \infty}\left\{\left(\frac{3 \beta^{3}}{8}\right)^{1 / 2} \frac{1}{\mathrm{Re}}+\sqrt{1+\frac{3 \beta^{3}}{8}} \frac{1}{\mathrm{Re}^{2}}\right\}
$$

and then calculating the ratio as $c_{\epsilon}(\alpha, \mathrm{Re}) / c_{\epsilon}^{\mathrm{fit}}(\alpha=0, \mathrm{Re})$, which reduces the systematic error. The drag coefficient parametrization (3) had been suggested in Ref. [23] as a crossover function from the viscous to the inertial regime. As seen from the inset of Fig. 1 the experimental data for $c_{\epsilon}(\alpha=0, \mathrm{Re})$ are well described by this fit. For the physi-

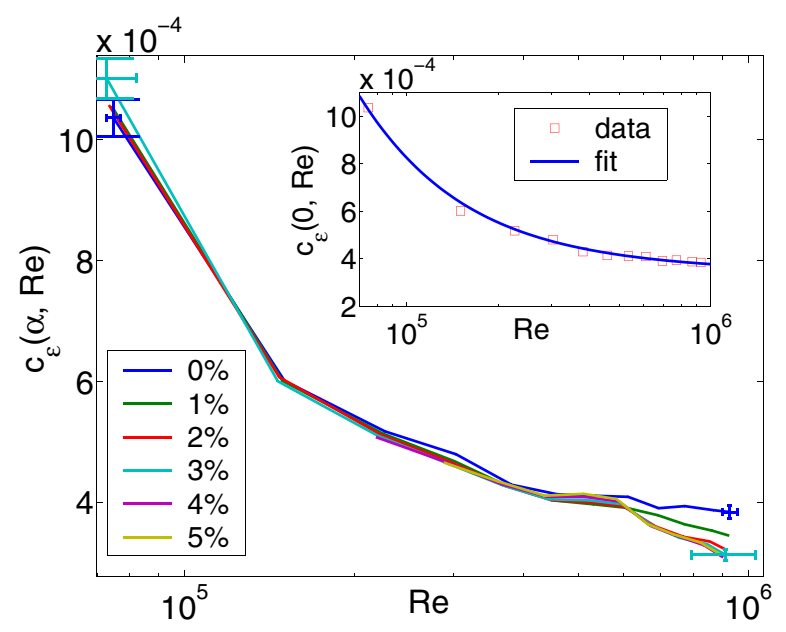

FIG. 1 (color online). The drag coefficient $c_{\epsilon}(\alpha, \mathrm{Re})$ for volume concentrations $\alpha$ up to 0.05 . The error bars in this and the subsequent figures result from an estimated relative error of $1 \%$ in the electronically controlled $\Omega$ and in $T$, and from an estimated absolute error of $0.5 \%$ for $\alpha$. For clarity, error bars are given only for the first and the last data points of each curve. The inset shows $c_{\epsilon}(\alpha=0, \operatorname{Re})$ for the single phase flow case $(\alpha=0)$ together with its fit Eq. (3) which nicely described the measured data. The fit parameters are $\beta=3000$ and $c_{\epsilon, \infty}=3.4 \times 10^{-4}$.

cal meaning of the fit parameters $\beta$ and $c_{\epsilon, \infty}$ we refer to Ref. [23].

From Fig. 2 we can identify two regimes: a regime up to $\operatorname{Re} \sim 6 \times 10^{5}$ with very little drag reduction of a few percent, and beyond that a regime of considerable drag reduction up to $20 \%$. This is considerable for the relatively small volume concentration and presumably cannot be explained as a bulk effect because the mass change is at most only $5 \%$. We therefore conclude that the bubbles indeed modify the turbulent boundary layers, as generally assumed [24].

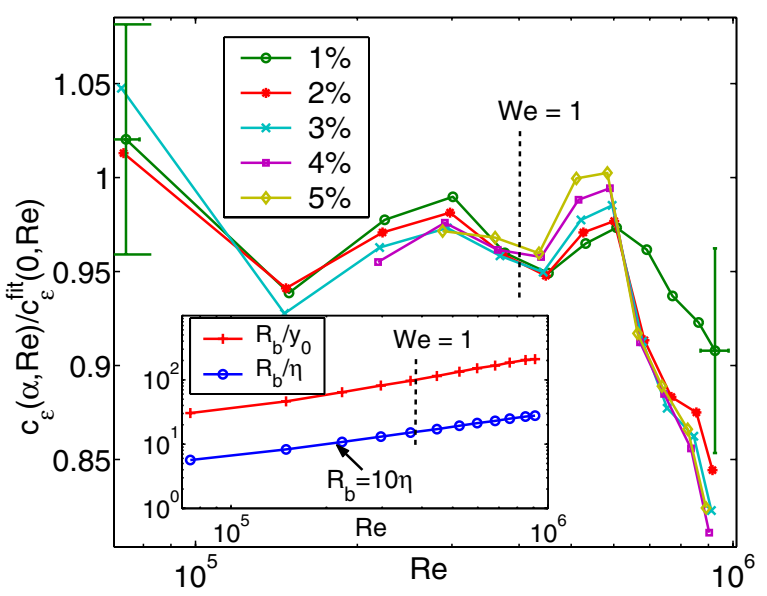

FIG. 2 (color online). Ratio $c_{\epsilon}(\alpha, \mathrm{Re}) / c_{\epsilon}^{\mathrm{fit}}(0, \mathrm{Re})$ vs Re for $0 \leq$ $\alpha \leq 0.05$. The inset shows the ratios $R / \eta$ (lower) and $R / y_{0}$ (upper) as a function of Re. 
To obtain some understanding of the crossover behavior observed in Fig. 2, we calculate how the Weber number $\mathrm{We}=\rho u^{12} R_{b} / \sigma$ depends on the Reynolds number. Here, We is based on the velocity fluctuations $u^{\prime}=i a \Omega$, where $i$ is the turbulent intensity. We thus get

$$
\mathrm{We}=\frac{\rho}{\sigma} \frac{\nu^{2} R_{b}}{(b-a)^{2}} i^{2} \mathrm{Re}^{2} \approx 2 \times 10^{-9} i^{2} \operatorname{Re}^{2},
$$

with $\quad \nu=8 \times 10^{-7} \mathrm{~m}^{2} / \mathrm{s}, \quad \rho=1000 \mathrm{~kg} / \mathrm{m}^{3}, \quad \sigma=$ $0.072 \mathrm{~N} / \mathrm{m}$ (all for $30^{\circ} \mathrm{C}$ ), and $R_{b} \approx 0.5 \mathrm{~mm}$ as it applies in our experiments. For the large $\operatorname{Re}=10^{5}-10^{6}$ it is $i \approx$ 0.06 [20]. Equation (4) thus predicts that the crossover from $\mathrm{We}<1$, where the bubbles are mainly spherical, to $\mathrm{We}>1$, where the bubbles are deformed, takes place at $\mathrm{Re}_{\mathrm{We}=1} \approx 4 \times 10^{5}$, very close to the observed transition in Fig. 2.

We also calculate the Kolmogorov length $\eta=\nu^{3 / 4} / \epsilon^{1 / 4}$ and $y_{0}$ and compare them with the typical bubble size; see the inset of Fig. 2. Note that the given length scales $\eta$ and $y_{0}$ can be only averaged length scales as their calculation is based on a global quantity (namely the torque); they, in fact, may depend on the position in the flow. In any case, one can judge from the inset of Fig. 2 that in the small Re regime $R_{b} \simeq 5 \eta$; i.e., the flow around the bubble is smooth and the bubble indeed can be more or less spherical. In the large Re regime, however, $R_{b} \gg 10 \eta$, and the bubbles can considerably deform and wobble.

We interpret the small $\mathrm{Re}$ regime with $\mathrm{We}<1$ as a regime in which the drag reduction mechanism suggested by Ferrante and Elghobashi [12] is dominant, namely, effective compressibility. For a given volume fraction this mechanism is most efficient for small bubbles. Since here our bubbles are large as compared to the tiny bubbles used in the Ferrante and Elghobashi simulations, we have only a small drag reduction effect. In the large Re regime with We $>1$, however, the dominant drag reduction effect seems to be the bubble deformability, as suggested by Lu et al. [13] and Sugiyama et al. [15]. For the large bubbles employed in this regime, the drag reduction indeed can be considerable, just as observed in our experiments.

To test this interpretation and to get more experimental information to either validate or falsify the theories on drag reduction, we repeated the torque measurements for turbulent Taylor-Couette flow, but now after having injected buoyant particles rather than bubbles. We took hollow glass spheres (Q-Cel hollow spheres from "PQ corporation" [26]) with a mean radius of $R_{p}=85 \mu \mathrm{m}$ and an effective density of $\rho_{p} / \rho_{l}=0.14$. The result for the drag coefficient $c_{\epsilon}$ is shown in Fig. 3. We find that for small Re the (small) drag reduction is within error bars the same as for the bubbly flow. For large Re, however, there is no drag reduction whatsoever for the buoyant particles. This finding is consistent with our above interpretation, as buoyant particles should show the drag reduction effect due to the effective compressibility [12], but not the one due to

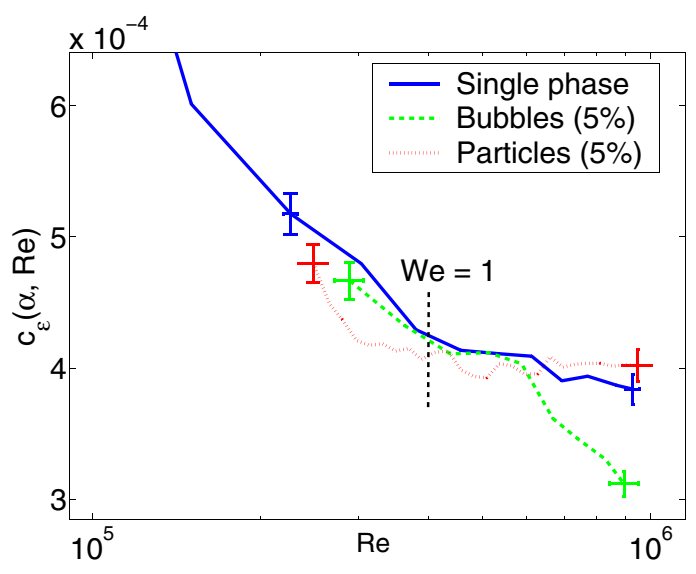

FIG. 3 (color online). Drag coefficient $c_{\epsilon}(\alpha, \mathrm{Re})$ vs $\mathrm{Re}$ for single phase flow (solid line), bubbly flow with $\alpha=0.05$ (dashed line), and buoyant particle flow with $\alpha=0.05$ (dotted line).

deformability $[13,15]$. For large Re the drag coefficient is even slightly enhanced (see Fig. 3). This may be in accordance with the predictions of $\mathrm{Lu}$ et al. [13] and Sugiyama et al. [15], or it may be an effect due to the finite inertia of the particles, which have distinctively nonzero mass $\left(\rho_{p} / \rho_{l}=0.14\right)$ and which could counteract the effective compressibility drag reduction effect. To what degree this will be the case can be answered only by additional numerical simulations with such particles and/or by additional experiments with particles of different effective density.

Finally, we address drag reduction for larger bubble concentrations up to $10 \%$. First of all, we note that strictly speaking the relation, Eq. (2), will no longer be valid at gas volume concentration of $5 \%$ and beyond. Nevertheless, we decided to present the ratio $c_{\epsilon}(\alpha, \operatorname{Re}) / c_{\epsilon}^{\text {fit }}(0, \mathrm{Re})$ as a function of $\alpha$ for $0 \leq \alpha<10 \%$ in Fig. 4, in order to give the reader an idea of what may be going on for large $\alpha$. Surprisingly, beyond $\alpha=5 \%$ the drag increases again.

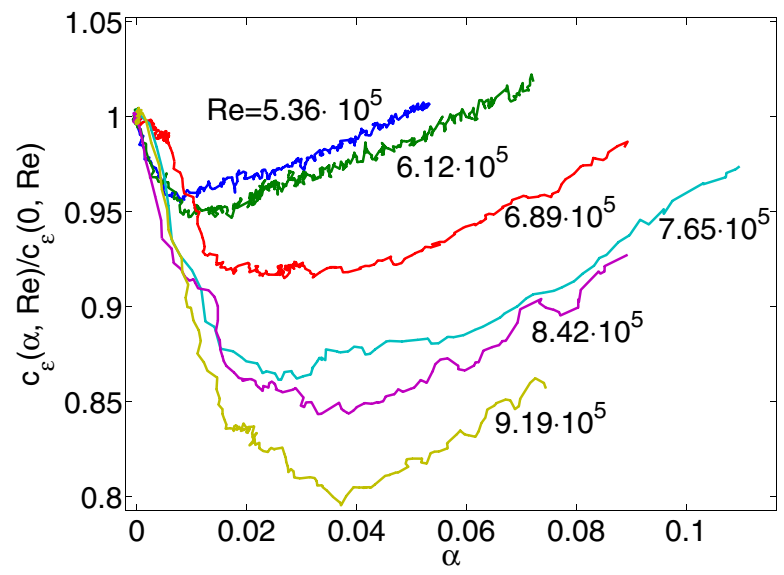

FIG. 4 (color online). Ratio $c_{\epsilon}(\alpha, \mathrm{Re}) / c_{\epsilon}^{\mathrm{fit}}(0, \mathrm{Re})$ vs $\alpha$ for various Re. 
From Eq. (1) it is seen that this result means that for a larger gas volume fraction an increasing torque $T$ is required to keep the inner cylinder running at constant angular velocity $\Omega$. A possible explanation of this effect is as follows: Because of the centrifugal acceleration of the water gas bubbles accumulate close to the inner cylinder. Once the gas fraction is too large (here beyond $5 \%$ ), the gas layer at the inner cylinder may prevent an efficient forcing of the liquid between the cylinders. An effective Reynolds number (e.g., the Taylor-Reynolds number $\operatorname{Re}_{\lambda}$ ) characterizing the degree of turbulence in the liquid may therefore be considerably smaller than suggested from $\operatorname{Re}=\Omega a(b-a) / \nu$. For fixed $\operatorname{Re}_{\lambda}$ the ratio $c_{\epsilon}\left(\alpha, \operatorname{Re}_{\lambda}\right) / c_{\epsilon}^{\mathrm{fit}}\left(0, \operatorname{Re}_{\lambda}\right)$ may, in fact, monotonously decrease as a function of $\alpha$. Unfortunately, presently we cannot measure $\operatorname{Re}_{\lambda}$ for our bubbly flow.

In conclusion, both the suggested effects for drag reduction in the bubbly flow seem to contribute, namely, effective flow compressibility [12] and bubble deformability $[13,15]$. The relative importance of the effects depends on the bubble size and flow Reynolds number. For smaller bubbles or smaller $\mathrm{Re}$ the former is more important (We $<1$ ), whereas the latter (We $>1$ ) is for larger bubbles or larger Re. In our experiments the crossover between the two regimes is around $\mathrm{Re} \approx 6 \times 10^{5}$, corresponding to $R_{b}=42 \eta=300 y_{0}$. Clearly, further experiments and simulations with various bubble and particle sizes and density ratios are motivated by these intriguing observations. Moreover, obtaining information on the radial bubble and particle distribution, on the bubble shape, and on the importance of bubble breakup would be useful. The closed flow Taylor-Couette cell seems to us to be a very suitable system for further analysis of drag reduction in the bubbly flow.

The authors thank Dan Lanterman for measuring the bubble size, Matthew Martin and Don Martin for technical assistance, and S. Elghobashi, Y. Matsumoto, Itamar Procaccia, Andrea Prosperetti, Shu Takagi, G. Tryggvason, and Leen van Wijngaarden for helpful discussions. The work is part of the research program of FOM, which is financially supported by NWO. It was also supported by the European Union (EU) under Contract No. HPRN-CT-2000-00162 and by the National Science Foundation of the USA.

*Electronic address: t.h.vandenberg@tnw.utwente.nl

†Electronic address: dpl@complex.umd.edu

*Electronic address: d.lohse@utwente.nl
[1] P. S. Virk, AIChE J. 21, 625 (1975).

[2] N. S. Berman, Ann. Rev. Fluid Dyn. 10, 47 (1978).

[3] R. Benzi, E. S. C. Ching, I. Procaccia, and N. Horesh, Phys. Rev. Lett. 92, 078302 (2004).

[4] V. S. L'vov, A. Pomyalov, I. Procaccia, and V. Tiberkevich, Phys. Rev. Lett. 92, 244503 (2004).

[5] N. K. Madavan, S. Deutsch, and C. L. Merkle, Phys. Fluids 27, 356 (1984).

[6] N. K. Madavan, S. Deutsch, and C. L. Merkle, J. Fluid Mech. 156, 237 (1985).

[7] C. Merkle and S. Deutsch, in Frontiers in Experimental Fluid Mechanics, Lecture Notes in Engineering Vol. 46, edited by M. G. el Hak (Springer, Berlin, 1989), p. 291.

[8] V. S. L'vov, A. Pomyalov, I. Procaccia, and V. Tiberkevich (to be published).

[9] Y. Kodama, A. Kakugawa, T. Takahashi, and H. Kawashima, Int. J. Heat Fluid Flow 21, 582 (2000).

[10] T. Takahashi et al., in Proceedings of the 74th General Meeting of SRI, 2000, http://www.srimot.go.jp/spd/drag/ drag2e.htm.

[11] J. Xu, M. R. Maxey, and G. E. Karniadakis, J. Fluid Mech. 468, 271 (2002).

[12] A. Ferrante and S. Elghobashi, J. Fluid Mech. 503, 345 (2004).

[13] J. Lu, A. Fernandez, and G. Tryggvason (to be published).

[14] K. Sugiyama, T. Kawamura, S. Takagi, and Y. Matsumoto, in Proceedings of the 4th Symposium on Smart Control of Turbulence, www.turbulence-control.gr.jp/PDF/ symposium/FY2002/Sugiyama.pdf.

[15] K. Sugiyama, T. Kawamura, S. Takagi, and Y. Matsumoto, in Proceedings of the 5th Symposium on Smart Conrol of Turbulence, www.turbulence-control.gr.jp/PDF/ symposium/FY2003/Sugiyama.pdf.

[16] S. O. Unverdi and G. Tryggvason, J. Comput. Phys. 100, 25 (1992).

[17] A. Einstein, Ann. Phys. (Leipzig) 19, 289 (1906).

[18] A. Acrivos, J. Rheol. (N.Y.) 39, 813 (1995).

[19] D. P. Lathrop, J. Fineberg, and H. S. Swinney, Phys. Rev. Lett. 68, 1515 (1992).

[20] G. S. Lewis and H.L. Swinney, Phys. Rev. E 59, 5457 (1999).

[21] T. H. van den Berg, C. Doering, D. Lathrop, and D. Lohse, Phys. Rev. E 68, 036307 (2003).

[22] F. Risso and J. Fabre, J. Fluid Mech. 372, 323 (1998).

[23] D. Lohse, Phys. Rev. Lett. 73, 3223 (1994).

[24] Note that in contrast the reduction of the energy dissipation rate observed in the numerical simulations of homogeneous turbulence [25] of course is a bulk effect. This is at most a $4 \%$ effect, comparable to the bubble volume concentrations in those simulations.

[25] I. Mazzitelli, D. Lohse, and F. Toschi, Phys. Fluids 15, L5 (2003); J. Fluid Mech. 488, 283 (2003).

[26] See www.pqcorp.com 